\title{
Dual-time point 18FDG-PET/CT imaging may be useful in assessing local recurrent disease in high grade bone and soft tissue sarcoma
}

\author{
Zhivka Dancheva, Pavel Bochev, Borislav Chaushev, Tsvetelina Yordanova, Aneliya Klisarova \\ Nuclear Medicine and Metabolic Therapy Department, Saint Marina University Hospital, Varna, Bulgaria
}

[Received 29 VI 2015; Accepted 10 IX 2015]

\begin{abstract}
BACKGROUND: Sarcomas comprise $1 \%$ of malignant tumors in adults but represent a significant diagnostic and therapeutic challenge. Molecular imaging with ${ }^{18} \mathrm{FDG}$ PET/CT is a powerful modality in oncology. Its use for initial assessment, evaluation of response to therapy and recurrent disease in most tumors is essential for therapeutic decisions. Its indication in sarcomas is still controversial. One of the indications for PET/CT in sarcomas is detection of recurrences. Nowadays magnetic resonance tomography (MRT) has a crucial role in identification of local recurrences in soft tissue and bone sarcoma. ${ }^{18}$ FDG-PET/CT may serve as a complementary method. Dual time point imaging (DTPI) has been studied for most tumors as a method for differentiating benign from malignant lesions. There is limited data on DTPI in sarcomas. Therefore we studied prospectively patients with suspected local recurrences in the treated area and used DTPI as a method for differentiating benign from malignant tissue. The aim of this study was to evaluate the ability of dual-time point PET/CT to enhance sensitivity, specificity, PPV, NPV and accuracy of ${ }^{18} \mathrm{FDG}$ PET/CT in high grade and low grade sarcomas.

MATERIAL AND METHODS: We conducted a dual-time PET/CT in 15 patients with suspected locally recurrent disease. The delayed scan was conducted on the 120th min in the suspected region. The interpretation of PET/CT was made both upon CT scan and metabolic scans. The percentage change over time per lesion was calculated (\%DSUV). The increase in SUVmax with $\%$ DSUV $>10 \%$ in the late scanning was considered as indicative for malignancy. We assessed the sensitivity, specificity, accuracy, positive and negative predicting value of the interpretation of PET/CT at $60 \mathrm{~min}$ and $120 \mathrm{~min}$. All of the patients were followed up for a period of 1-3 years after our examination, either with histologic results, or with an MRT scans.

RESULTS: The received sensitivity, specificity and accuracy of ${ }^{18} \mathrm{FDG}$ PET/CT interpretation at 120 min in high grade sarcomas were respectively $100 \%, 80 \%$ and $89 \%$. By comparison, in low grade tumors at 120 min scan, these parameters were $50 \%$, $75 \%$ and $66 \%$.

CONCLUSION: These preliminary data suggests that dual-time imaging in sarcomas improves sensitivity and accuracy in identification of local recurrent disease in high grade sarcomas and have limited role in low grade sarcomas. Further research is necessary to confirm these results.
\end{abstract}

KEY words: soft tissue, bone sarcomas, dual- time-point imaging, ${ }^{18} \mathrm{FDG}-\mathrm{PET} / \mathrm{CT}$, local recurrence

Nuclear Med Rev 2016; 19, 1: 22-27

\section{Background}

Sarcomas comprise $1 \%$ of malignant tumors in adults. They are a large heterogeneous group of entities with different biologi-

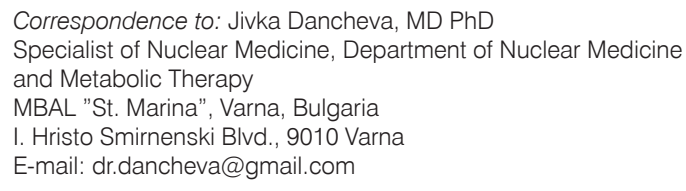

cal behavior and the clinical diagnosis of such lesions can be very difficult. Molecular imaging with ${ }^{18}$ FDG PET/CT (fluoro-deoxyglucose-positron emission tomography with computed tomography) is a powerful modality in oncology. Its use for initial assessment, staging, restaging, evaluation of response to therapy and recurrent disease in most tumors is essential for therapeutic decisions. It has been utilized as an alternative pretreatment imaging method for both soft tissue and bone sarcomas [1, 2]. Another use of ${ }^{18} \mathrm{FDG}-\mathrm{PET} / \mathrm{CT}$ is evaluation of response to chemotherapy. Definitive treatment of sarcoma is surgical resection. However, after surgical 
removal, $40 \%$ to $60 \%$ of the patients will develop local or distant recurrence. Detection of local recurrent disease is still a controversial indication for PET/CT in sarcomas. Nowadays magnetic resonance tomography (MRT) has a crucial role in identification of local recurrences in soft tissue and bone sarcoma. ${ }^{18}$ FDG-PET/CT may serve as a complementary method, searching for metabolic activity in treated regions. The accumulation and metabolism of ${ }^{18} \mathrm{FDG}$ is dependent on many factors. Glucose-6-phosphatase, hexokinase and GLUT transporters appear to be most important. Dual-time-point imaging (DTPI) has been studied for most tumors as a method for differentiating benign from malignant lesions. There is limited data on DTPI in sarcomas. Therefore we studied patients with suspected local recurrences in the treated area prospectively using DTPI as a method for differentiating post treatment inflammation vs. local malignancy.

Our aim was to study the ability of dual-time-point PET/CT to enhance sensitivity, specificity, PPV, NPV and accuracy of ${ }^{18} \mathrm{FDG}$ $\mathrm{PET} / \mathrm{CT}$ in high grade and low grade sarcomas.

\section{Materials and methods}

\section{Patients}

For a period of six years (2009-2015), we examined 15 patients with suspected local recurrent disease. The patients were two women and thirteen men, from 27 to 78 years old. The final verification was made by histologic results either with MRT scans and all of them were followed up clinically for a period of 1-6 years after our examination.

\section{Inclusion parameters}

All of the patients had previously locally treated sarcoma and imaging findings suspicious for residual or recurrent disease from other imaging modalities. We performed 120 min scan in those patients who have been treated locally for sarcoma (excision, radiotherapy or alloplastic joint replacement) and had difficult for interpretation PET/CT 60 min scans. We conducted a second scan if the local SUVmax was 2.0 or higher but was not sufficient to be interpreted as a local recurrent disease. We included patients with soft tissue and bone sarcomas of different grades. The number of various histological subtypes of sarcomas according to the AJCC classification and their grading are summarized in Table 1. There were 8 patients with high grade tumors and 6 patients with low grade tumors.

Table 1. Type and grade of sarcomas in the examined patients

$\begin{array}{llcc}\text { Type of sarcoma } & \mathbf{n} & \text { Low grade }(\mathbf{n}) & \text { High grade }(\mathbf{n}) \\ \text { Hondrosarcoma } & 1 & 3 & 1 \\ \text { Liposarcoma } & 7 & & 4 \\ \text { Ewing sarcoma } & 1 & 1 & 1 \\ \text { Malignant fibrous histiocytoma } & 1 & 1 & 2 \\ \text { Leiomyosarcoma } & 2 & 1 & \\ \text { Sinovial sarcoma } & 1 & 1 & \\ \text { Fibrosarcoma } & 1 & 1 & \end{array}$

\section{Methods}

The examination was held on Gemini TF PET/CT, Philips, equipped with 16 slice CT. We performed low-dose CT. The PET/CT scan was held at two time points, at $60 \mathrm{~min}$ and at $120 \mathrm{~min}$. The early scan was obtained from the skull base to mid-thigh, whereas the second scan was acquired only for the area of interest.

PET/CT imaging was performed in patients three months after radiotherapy and one month after surgery or chemotherapy. All of the patients fasted for at least $6 \mathrm{~h}$ before the examination to ensure standardized glucose metabolism. At the time of ${ }^{18} \mathrm{FDG}$ administration, fasting plasma glucose values were lower than $150 \mathrm{mg} / \mathrm{dL}$ in all patients. Depending on the patients' weight, a dose of $185-555 \mathrm{mBq}$ was administrated through a catheter inserted into an antecubital vein

\section{Interpretation}

The interpretation of PET/CT was made upon CT scan, metabolic scan and fused scans. We used SUVmax-based quantification for differentiation of benign from malignant lesions. Studies have demonstrated significant variability of SUVmax values in test-retest measurements, ranging from 6 to 10 percent [3] with increase in variability with higher SUVmax values. The percentage change over time per lesion was calculated (\%DSUV). Zuang et al. published similar results in DTPI with PET/CT in a variety of tumors. The average SUV of the lesions in known cancer patients increased from 3.96 at the first time point to 4.72 at the second time point $(+19.2 \% \pm 9.6 \% ; n=31 ; P<0.001)$ [4]. As reproducibility of SU$V m a x$ is subject to significant variation, we accepted as indicative for malignancy the increase in SUVmax with \%DSUV $>10 \%$ in the late scanning. The sensitivity, specificity, accuracy; PPV and NPV were estimated in all of the patients and in the two groups - with low and with high grade tumors.

\section{Results}

We assessed the sensitivity, specificity, positive (PPV), negative predictive value (NPV) and accuracy in all of the cases at 60 and 120 min and separately in high grade and low grade sarcomas at $120 \mathrm{~min}$. Results are listed in Tables 2 and 3. The evaluation was made for PET/CT interpretations at $60 \mathrm{~min}$ and at $120 \mathrm{~min}$.

True positive results at 120 min were obtained in $3 \mathrm{pa}$ tients with high grade tumors and in one patient with low grade tumor (Figure 1). True negative results were obtained in

Table 2. True positive, true negative, false positive and false negative results in patients at $60 \mathrm{~min}$ and $120 \mathrm{~min}$, and in high and low grade sarcomas at $120 \mathrm{~min}$

\begin{tabular}{|c|c|c|c|c|}
\hline & \multicolumn{2}{|c|}{ All patients } & $\begin{array}{l}\text { High grade } \\
\text { sarcomas }\end{array}$ & \multirow{2}{*}{$\begin{array}{c}\text { Low grade } \\
\text { sarcomas } \\
120 \mathrm{~min}\end{array}$} \\
\hline & $60 \mathrm{~min}$ & $120 \mathrm{~min}$ & $120 \mathrm{~min}$ & \\
\hline TP & 2 & 4 & 3 & 1 \\
\hline TN & 5 & 8 & 5 & 3 \\
\hline FP & 5 & 2 & 1 & 1 \\
\hline FN & 3 & 1 & 0 & 1 \\
\hline
\end{tabular}


Table 3. Results of the sensitivity, specificity, PPV, NPV and accuracy in all of the patients and in two groups of patients: with high grade and with low grade sarcomas

\begin{tabular}{lcccc} 
& \multicolumn{2}{c}{ All patients } & $\begin{array}{c}\text { High grade } \\
\text { sarcomas }\end{array}$ & $\begin{array}{c}\text { Low grade } \\
\text { sarcomas }\end{array}$ \\
\cline { 2 - 3 } & $60 \mathrm{~min}$ & $120 \mathrm{~min}$ & $120 \mathrm{~min}$ & $120 \mathrm{~min}$ \\
\hline Sensitivity & $40 \%$ & $80 \%$ & $100 \%$ & $50 \%$ \\
Specificity & $50 \%$ & $80 \%$ & $83 \%$ & $75 \%$ \\
PPV & $29 \%$ & $89 \%$ & $75 \%$ & $50 \%$ \\
NPV & $63 \%$ & $89 \%$ & $100 \%$ & $75 \%$ \\
Accuracy & $47 \%$ & $80 \%$ & $89 \%$ & $67 \%$
\end{tabular}

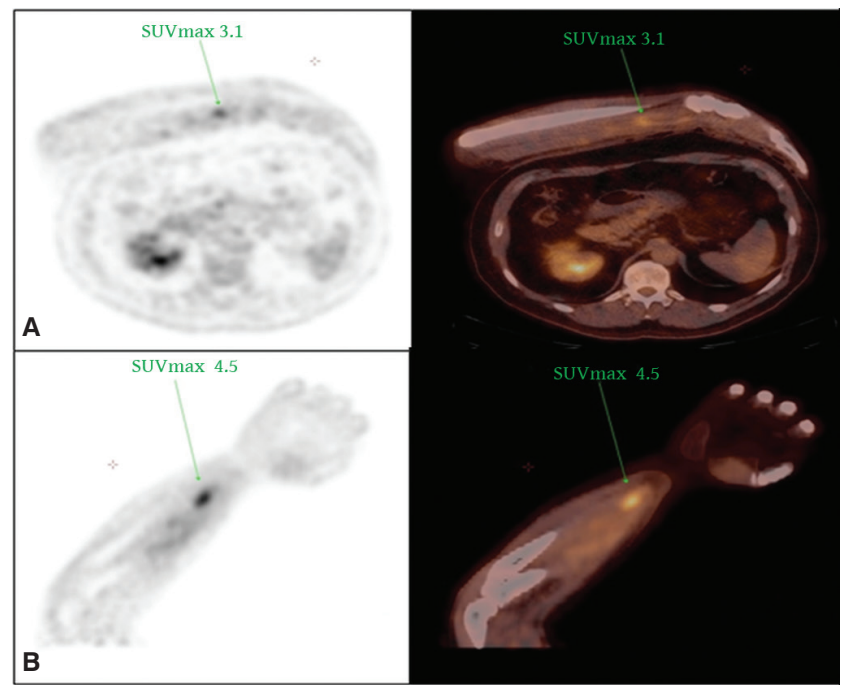

Figure 1. A patient with MFH of the right forearm: A. Equivocal $60 \mathrm{~min}$ scan with SUVmax in the operation area of 3.1 ; B. The 120 min scan was positive with SUVmax 4.5 , later confirmed by histology as a recurrent tumor

5 patients with high grade tumors and 3 patients with low grade tumors (Figure 2).

The 120 min scan was not helpful to reveal two of the false positives at 60 min PET/CT scan. (Figure 3 and 4) These two cases, one in high grade and one in low grade group, significantly raised their SUVmax at $120 \mathrm{~min}$. Both of the cases were with chronic osteomyelitis after local treatment — one after the tumor excision and the other after excision and radiotherapy.

There were three cases of false negative PET/CT interpretations for recurrent disease at $60 \mathrm{~min}$ scan because of low to medium SUVmax 2.8-3.1. The three of them became positive at the $120 \mathrm{~min}$ scan with SUVmax increase of 39-42\%. All of them were high grade tumors. There was only one case with low grade tumor and false negative result at $120 \mathrm{~min}$. The $60 \mathrm{~min}$ scan was correctly interpreted as positive. It was with inhomogeneous uptake in the treated area and SUVmax 3.1 at 60 min scan suspicious for recurrent disease. SUVmax did not increase at $120 \mathrm{~min}$. and was interpreted falsely as negative. That patient had many local recurrences followed by local excisions. After the histology result it was proved to be tumor tissue.

The total sensitivity of the 60 min PET/CT scan for determination of a recurrent disease was only $40 \%$. By the 120 min scan

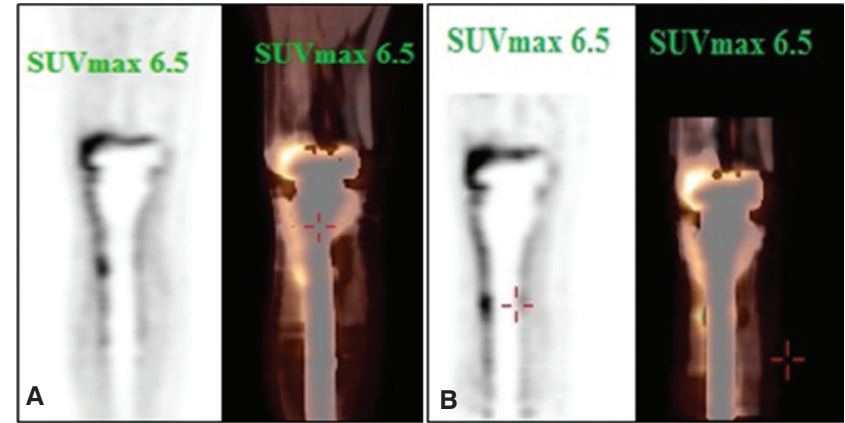

Figure 2. A patient with high grade chondrosarcoma five years after chemotherapy and tumor excision with alloplastic replacement of the right knee joint; A. (Early scan) Although the high SUVmax at 60 min scan - 6.5, at the late scan (B) this value did not change true negative result. This person was followed up for 5 years without recurrent disease

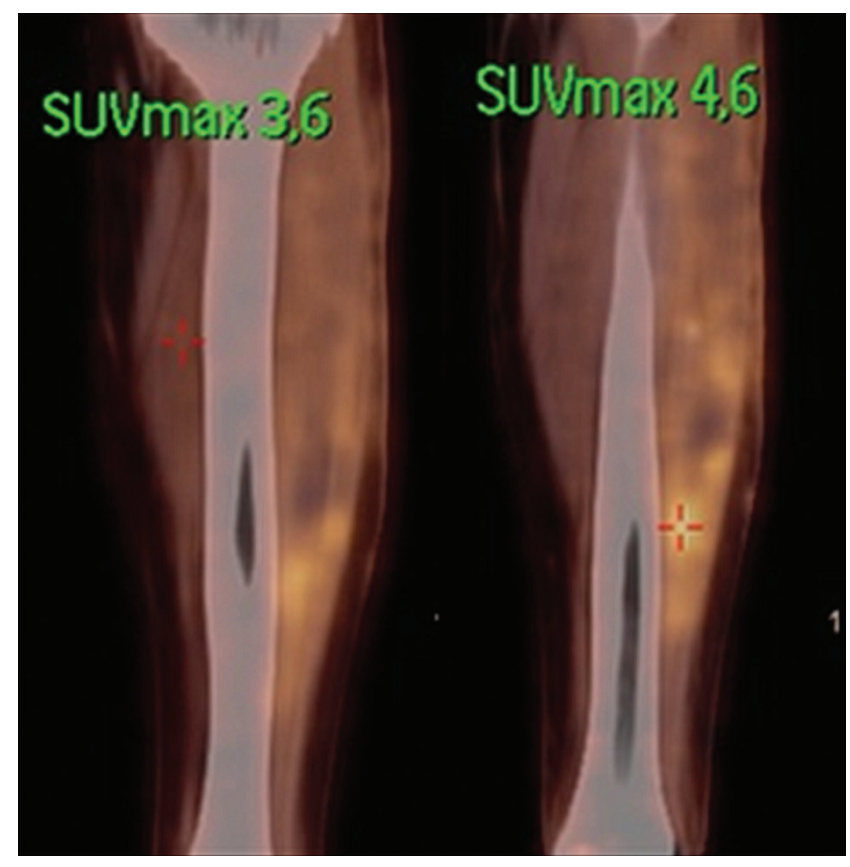

Figure 3. A patient with low grade fibrosarcoma three months after tumor excision and fibular resection. The 120 min scan showed $27.8 \%$ increase in activity in the primary tumor area. The result was proved false positive, as the patient had no tumor tissue but only inflammatory cells

methodology, we managed to determine the recurrent disease in all of the patients with high grade sarcomas and only in 50\% in those with low grade tumors. The specificity of the method was better for high grade sarcomas $83 \%$. The specificity was reduced because of the false positive results in two patients with chronic osteomyelitis.

The PPV was better in patients with high grade sarcomas $-75 \%$ vs $50 \%$ in low grade sarcomas. The NPV was better in the late study; in high grade sarcomas it was $100 \%$, revealing all of the patients without disease.

Accuracy in identifying recurrent disease in high grade sarcomas, using the dual-time-point PET/CT scan was $89 \%$, vs. $67 \%$ in low grade sarcomas. 


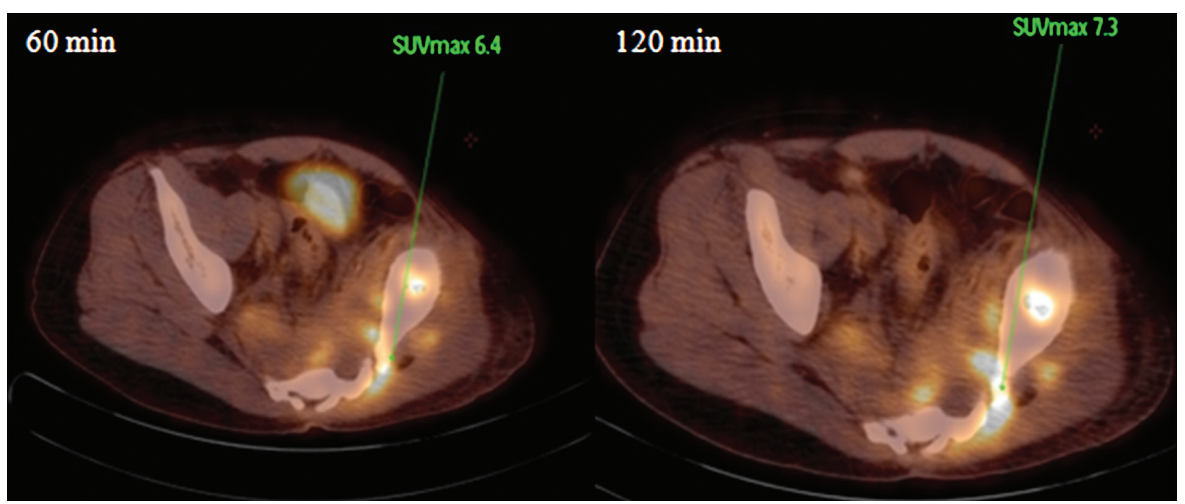

Figure 4. A patient with Ewing sarcoma 18 years after operation and radiotherapy. A fracture of the left iliac bone is seen. The SUVmax at 120 min scan increased by $14.1 \%$ - a false positive result, due to chronic osteomyelitis

\section{Discussion}

PET/CT was studied as a helpful instrument for initial assessment in sarcoma, but also for evaluation of response to therapy [5] and detection of recurrences in STS (soft tissue sarcoma) and BS (bone sarcoma). During the initial workup of sarcomas, MRT provides better description and definition of the local extent of the tumor. PET/CT has been found to be useful in prognostication, grading and assessing response to chemotherapy $[2,6,7]$. SUVmax has been shown to correlate with tumor grade and progression [8, 9]. Lodge et al. [10] noted that in high grade sarcomas, the maximal ${ }^{18} \mathrm{FDG}$ uptake is reached at $4 \mathrm{~h}$ whereas benign lesions achieve such values within $30 \mathrm{~min}$. False negative findings were often found in low grade sarcomas.

We used SUVmax-based quantification for revealing malignant lesions in the treated area of the primary tumor. Studies have demonstrated significant variability of SUVmax values in test-retest measurements, ranging from 6 to 10 percent $[3,4]$ with increase in variability with higher SUVmax values. Evaluating the DTPI value in lung nodules, Matthies et al concluded that dual-time-point scanning with a threshold value of $10 \%$ increase between scan 1 and scan 2 reached a sensitivity of $100 \%$ with a specificity of $89 \%$ [11]. We also accepted that a change of SUVmax higher or equal to $10 \%$ was significant for interpretation of malignancy at the 120 min scan.

The logic behind delayed ${ }^{18}$ FDG PET/CT imaging is based on the known fact that most types of malignant cells have significantly increased ratios of hexokinase to glucose-6-phosphatase activities, which allow ${ }^{18} \mathrm{~F}$-deoxyglucose-6-phosphate to accumulate to a much higher level over time than in the normal cells. There is insufficient data on the role of PET/CT in patients with suspected recurrent sarcoma after local therapy. In rhabdomyosarcoma PET/CT was found useful for detecting recurrent disease in treated patients. [12] It may be useful for initial staging too because of the possibility of nodal metastases and unknown distant metastases. In these patients management intent may change from curative to palliative, thereby sparing patients the morbidity associated with non-beneficial aggressive treatment. A few articles are focused on the performance of DTPI and delayed time-point imaging in assessing residual/recurrent lesions after therapy in breast cancer and in head and neck cancer $[13,14]$. PET/CT should avoid unnecessary biopsy through ruling out residual or recurrent active disease. Patient outcomes may be improved through reduced treatment morbidity.

The major value of DTPI is the increased sensitivity due to continued clearance of background activity and continued ${ }^{18} \mathrm{FDG}$ accumulation in malignant lesions. In order to homogenize the groups of patients, we divided them into two groups - with low and high grade sarcomas. In our investigation, we had only one false negative result at $120 \mathrm{~min}$ in low grade sarcoma, and no false negatives in high grade sarcomas. All of the false negative high grade sarcomas at 60 min turned to true positives at 120 min scan, which was the most important achievement in our investigation. The yielded sensitivity at 120 min increased from $40 \%$ to $80 \%$ for all cases. In high grade sarcomas group we managed to reach $100 \%$ sensitivity at $120 \mathrm{~min}$, which was significantly better result than in the low grade sarcomas group only $50 \%(p=0.034)$. Tumor heterogeneity, the small size of the recurrent tumor and low grade tumor (low metabolic activity) may contribute to variable performance of DTPI ${ }^{18}$ FDG PET/CT imaging $[15,16]$. Tumor heterogeneity is based on variations in tumor cells (tumor genotype, differentiation) or in the environment (stromal matrix and tumor location, tissue vascularity and tumor size, and immune status, etc.). Tumor heterogeneity in sarcomas is very often. In our cases the only false negative result at 120 min was a patient with many recurrent episodes and treatments. He had a low grade tumor. This suggested the limited value of DTPI for the characterization of non-18FDG-avid tumors which was reported also in pancreatic cancer with low SUVmax [17].

Dual-time-point imaging has been used to increase specificity of ${ }^{18} \mathrm{FDG}$ PET for discriminating benign from malignant lesions. That is exceptionally important in the treated area, where operations and local radiotherapy may cause inflammatory comp-point ${ }^{18} \mathrm{FDG}$-PET imaging in this regard, and the technique has been studied in a wide variety of malignancies, including those of head and neck [18], lung [11], breast [19-21] gallbladder [22], cervix [23] and glioma [24]. In all of them authors concluded that delayed ${ }^{18}$ FDG-PET imaging is more helpful than early scans for evaluating malignant lesions. However, exceptions exist, and some studies have demonstrated significant overlap of ${ }^{18} \mathrm{FDG}$ uptake patterns between benign and malignant lesions on delayed time-point images. In many of these studies, dual time point PET improved both the sensitivity and the specificity of PET/CT. This can be explained by two factors: while continuing increased uptake in the malignant lesions allows differentiation from benign lesions with higher specificity, increased 
lesion-to-background leads to higher sensitivity with this method. In the majority of normal tissues (the blood pool, liver, spleen, lungs, pancreas, inguinal nodes, and skeletal muscles), ${ }^{18} \mathrm{FDG}$ uptake decreases significantly from 1 to $3 \mathrm{~h}$ after radiotracer injection, although SUV remains stable in some tissues (parotid gland, thyroid gland, and prostate gland) and increases in other tissues (myocardium and bone marrow) [25].

The specificity of this method in our study was increase from $50 \%$ in all cases to $80 \%$ at 120 min imaging. In high grade tumors group our study reached specificity $83 \%$ at 120 min scan (60 min scan specificity in this group was $67 \%$ ). The specificity in low grade tumors group also was better at 120 min scan $(75 \%$ vs $25 \%$ in early scan). We could not reduce two of the false positive results with chronic post treatment inflammation. All of the true negatives in both groups at 60 min scan remained TN at 120 min scan. Our method was useful to reveal three of the true negatives: two cases after tumor excision and one with alloplastic joint and high SUVmax at 60 min scan. At the late scan the SUVmax did not change or lowered, with 19\% in a low grade liposarcoma.

There are numerous reports in the literature of false-positive findings on ${ }^{18} \mathrm{FDG}$ PET imaging due to granulomas or active inflammation. The granulomas may arise many years after the initial trauma or procedure in the form of tumor-like soft-tissue masses [26-28]. Multiple studies have indicated that lesions of active inflammation and infection may have higher ${ }^{18} \mathrm{FDG}$ activity on DTPI, similar to that in malignant lesions, thus resulting in suboptimal diagnostic performance of DTPI [29-31]. There were two false positive cases among our patients, both with osteomyelitis, which remained false positive at late scans. Respectively, their SUVmax values were: SUVmax (60 min) 3,6/SUVmax (120) 4.6 and SUVmax (60 min) 6,4/SUVmax (120) 7.3. This was a change in the SUVmax with respectively $27.8 \%$ and $14.1 \%$. This results in future indicates the need for more careful selection of the patients for PET/CT scan and necessity of histologic confirmation after PET/CT positive findings. On the other hand, our methodology was able to resolve the problem with the metabolic active lesions at 60 min scan in three cases with active inflammation. One of the cases was a patient after alloplastic joint replacement with SUVmax (60 min) 6.5. This value stayed the same at $120 \mathrm{~min}$ scan and was interpreted as a negative for recurrent disease, lately confirmed by 3-year follow up. Another case was a patient with low grade tumor excision in whom SUVmax (60 min) was 4.2. After the late scan it decreased by $19 \%$ - SUVmax (120 min) 3.4. The third case also had a stable SUVmax value at the early and delayed scan (SUVmax 2.9) and was interpreted as negative after the 120 min scan. It was a patient with a high grade tumor. All of these data showed that increased ${ }^{18} \mathrm{FDG}$ uptake on delayed time-point images should not be used as the sole criterion in interpreting ${ }^{18}$ FDG PET studies. Correlation with other imaging findings, as well as the patient's history, should also be taken into consideration. And the late scans could improve the specificity both in high grade and low grade sarcomas.

The PPV was very low at 60 min scan for all patients - 29\%, which was because of too many complicated cases with false positive scans at that time point. Our methodology managed to increase the PPV — from $29 \%$ to $67 \%$ at 120 min scan, resolving 3 of 5 cases with false positive and all three false negative 60 min scans (changing the classification into true positives). In high grade sarcomas group the gained PPV was $75 \%$ vs. $50 \%$ in low grade tumors group.
In different studies the NPV of ${ }^{18} \mathrm{FDG}$-PET/CT in revealing recurrent disease varies from 75 to $100 \%$. Other studies did not separate the subjects into groups according to their tumor differentiation. In our investigation NPV was $100 \%$ in high grade sarcomas at 120 min scan compared with $63 \%$ at 60 min scan and $89 \%$ at 120 min scan in all patients. In low grade tumors the NPV was $75 \%$. At 120 min scan three of the false positive subjects turned into true negatives, because the SUVmax value did not change or got lower. Other false positives at 60 min were two cases with inflammatory complications. Excluding those two subjects, our methodology was useful for revealing all of the patients without recurrent disease.

Accuracy of the method also improved the values in the whole group - $47 \%$ at 60 min scans and $80 \%$ at 120 min scans. It was again better, although not significantly, in high grade tumors group $89 \%$ vs. $67 \%$ in low grade tumors.

\section{Conclusion}

Most malignant (and some inflammatory) lesions have increased ${ }^{18} \mathrm{FDG}$ uptake on delayed time-point images. Our study suggests that dual- time 18FDG-PET/CT in sarcomas might have a valuable role in detecting local recurrent disease in high grade sarcomas, as the sensitivity of the method gained significantly better results than in low grade sarcomas group at the same time point. The specificity, PPV and NPV are better in patients without late radiation and surgery complications. Accuracy was also better in high grade sarcomas. These results suggest that we must carefully select the patients for late PET/CT scan and have thorough information about current inflammations in the treated area.

\section{Conflicts of interest}

The authors declare that they have no conflicts of interest.

\section{References}

1. Völker T, Denecke T, Steffen I et al. Positron emission tomography for staging of pediatric sarcoma patients: results of a prospective multicenter trial. J Clin Oncol 2007; 25: 5435-5441.

2. Schuetze SM. Utility of positron emission tomography in sarcomas. Curr Opin Oncol 2006; 18: 369-373.

3. Wahl RL et al. From RECIST to PERCIST: Evolving Considerations for PET response criteria in solid tumors. J Nucl Med 2009; 50 (Suppl. 1): 122S-150S.

4. Zhuang $\mathrm{H}$, Pourdehnad M, Lambright ES et al. Dual time point 18F-FDG PET imaging for differentiating malignant from inflammatory processes. J Nucl Med 2001; 42: 1412-1417.

5. Piperkova E, Mikhaeil M, Mousavi A et al. Impact of PET and CT in PET/CT studies for staging and evaluating treatment response in bone and soft tissue sarcomas. Clin Nucl Med 2009; 34: 146-150.

6. Eary JF, O'Sullivan F, Powitan Y et al. Sarcoma tumor FDG uptake measured by PET and patient outcome: a retrospective analysis. Eur J Nucl Med Mol Imaging 2002; 29: 1149-1154.

7. Schuetze SM, Rubin BP, Vernon C et al. Use of positron emission tomography in localized extremity soft tissue sarcoma treated with neoadjuvant chemotherapy. Cancer 2005; 103: 339-348.

8. Folpe AL, Lyles RH, Sprouse JT, Conrad EU 3rd, Eary JF. (F-18) fluorodeoxyglucose positron emission tomography as a predictor of pathologic grade and other prognostic variables in bone and soft tissue sarcoma. Clin Cancer Res 2000; 6: 1279-1287.

9. Schwarzbach MH, Dimitrakopoulou-Strauss A, Willeke F at al. Clinical value of [18-F]] fluorodeoxyglucose positron emission tomography imaging in soft tissue sarcomas. Ann Surg 2000; 231: 380-386. 
10. Lodge MA, Lucas JD, Marsden PK, Cronin BF, O'Doherty MJ, Smith MA. A PET study of 18 FDG uptake in soft tissue masses. Eur J Nucl Med 1999; 26: 22-23.

11. Matthies A, Hickeson M, Cuchiara A, Alavi A. Dual-time-point 18F-FDG PET for the evaluation of pulmonary nodules. J Nucl Med 2002; 43: 871-875.

12. Sharma P, Khangembam BC, Suman KC et al. Diagnostic accuracy of 18F-FDG PET/CT for detecting recurrence in patients with primary skeletal Ewing sarcoma. Eur J Nucl Med Mol Imaging 2013; 40: 1036-1043.

13. Suga K, Kawakami Y, Hiyama A, Matsunaga N. Differentiation of FDG-avid loco-regional recurrent and compromised benign lesions after surgery for breast cancer with dual-time point F-18-fluorodeoxy-glucose PET/CT scan. Ann Nucl Med 2009; 23: 399-407.

14. Kubota K, Yokoyama J, Yamaguchi K et al. FDG-PET delayed imaging for the detection of head and neck cancer recurrence after radio-chemotherapy: comparison with MRI/CT. Eur J Nucl Med Mol Imaging 2004; 31: 590-595.

15. Basu S, Kwee TC, Gatenby R, Saboury B, Torigian DA, Alavi A. Evolving role of molecular imaging with PET in detecting and characterizing heterogeneity of cancer tissue at the primary and metastatic sites, a plausible explanation for failed attempts to cure malignant disorders. Eur J Nucl Med Mol Imaging 2011; 38: 987-991.

16. Asselin M-C, O'Connor JPB, Boellaard R, Thacker NA, Jackson A. Quantifying heterogeneity in human tumours using MRI and PET. Eur J Cancer 2012; 48: 447-455.

17. Higashi T, Saga T, Nakamoto $Y$ et al. Relationship between retention index in dual-phase (18)FFDG PET, and hexokinase-II and glucose transporter-1 expression in pancreatic cancer. J Nucl Med 2002; 43: 173-180.

18. Hustinx R, Smith RJ, Benard F et al. Dual time point fluorine-18 fluorodeoxyglucose positron emission tomography: a potential method to differentiate malignancy from inflammation and normal tissue in the head and neck. Eur J Nucl Med 1999; 26: 1345-1348.

19. Kumar R, Loving VA, Chauhan A, Zhuang H, Mitchell S, Alavi A. Potential of dual-time-point imaging to improve breast cancer diagnosis with $18 \mathrm{~F}-\mathrm{FDG}$ PET. J Nucl Med 2005; 46: 1819-1824.
20. Mavi A, Urhan M, Yu JQ et al. Dual time point 18F-FDG PET imaging detects breast cancerwith high sensitivity and correlates well with histologic subtypes. J Nucl Med 2006; 47: 1440-1446.

21. Boerner AR, Weckesser $\mathrm{M}, \mathrm{Herzog} \mathrm{H}$ et al. Optimal scan time for fluorine-18 fluorodeoxyglucose positron emission tomography in breast cancer. Eur J Nucl Med 1999; 26: 226-230.

22. Nishiyama Y, Yamamoto Y, Fukunaga K et al. Dual-time-point 18F-FDG PET for the evaluation of gallbladder carcinoma. J Nucl Med 2006; 47: 633-638.

23. Ma SY, See LC, Lai CH et al. Delayed 18F-FDG PET for detection of paraaortic lymph node metastases in cervical cancer patients. J Nucl Med 2003; 44: 1775-1783.

24. Spence AM, Muzi M, Mankoff DA et al. 18F-FDG PET of gliomas at delayed intervals: improved distinction between tumor and normal gray matter. J Nucl Med 2004; 45: 1653-1659.

25. Cheng G, Alavi A, Lim E, Werner TJ, Del Bello CV, Akers SR. Dynamic changes of FDG uptake and clearance in normal tissues. Mol Imaging Biol. 2012. doi: 10.1007/s11307-012-0600-0.

26. Cheng G, Torigian DA, Zhuang $\mathrm{H}$, Alavi A. When should we recommend use of dual time-point and delayed time-point imaging techniques in FDG PET? Eur J Nucl Med Mol Imaging 2013; 40: 779-787.

27. Tenconi S, Luzzi L, Paladini P et al. Pleural granuloma mimicking malignancy 42 years after slurry talc injection for primary spontaneous pneumothorax. Eur Surg Res 2010; 44: 201-203.

28. Miyake KK, Nakamoto $Y$, Mikami $Y$ et al. F-18 FDG PET of foreign body granuloma: pathologic correlation with imaging features in 3 cases. Clin Nucl Med 2010; 35: 853-857

29. Sathekge MM, Maes A, Pottel H, Stoltz A, van de Wiele C. Dual time-point FDG PET-CT for differentiating benign from malignant solitary pulmonary nodules in a TB endemic area. S Afr Med J 2010; 100: 598-601.

30. Umeda $Y$, Demura $Y$, Morikawa $M$ et al. Prognostic value of dual-time-point 18Ffluorodeoxyglucose positron emission tomography in patients with pulmonary sarcoidosis. Respirology 2011; 16: 713-720.

31. Cloran FJ, Banks KP, Song WS, Kim Y, Bradley YC. Limitations of dual time point PET in the assessment of lung nodules with low FDG avidity. Lung Cancer 2010; 68: 66-71. 